\title{
Optimum multistage genomic selection in dairy cattle
}

\author{
V. Börner, F. Teuscher, and N. Reinsch ${ }^{1}$ \\ Leibniz Institute for Farm Animal Biology, Research Unit Genetics and Biometry, 18196 Dummerstorf, Germany
}

\begin{abstract}
The availability of different single nucleotide polymorphism (SNP) chips and the development of imputation algorithms allow for multistage dairy cattle breeding schemes applying various genomic selection strategies. These SNP genotypes yield genomically estimated breeding values (GEBV) with different accuracies at different costs. Thus, the optimum allocation of investments to different selection paths and strategies to maximize the genetic gain per year $\left(\Delta G_{a}\right)$ and its sensitivity to changes in cost and accuracies of GEBV is of great interest. This is even more relevant under the constraints of limited financial resources. With deterministic methods, optimum multistage breeding plans maximizing $\Delta \mathrm{G}_{\mathrm{a}}$ were identified in which selection could take place on GEBV derived from high-density $\left(\mathrm{GEBV}_{\mathrm{HD}}\right)$ and low-density $\left(\mathrm{GEBV}_{\mathrm{LD}}\right) \mathrm{SNP}$ genotypes. To account for the uncertainty of cost and accuracies of GEBV, these parameters were varied in a semi-continuous manner. Overall breeding costs were limited to the crucial expenses of a traditional breeding program with 50 progeny-tested young bulls per year. Results clearly show that, in an optimal selection strategy, selection on $\mathrm{GEBV}_{\mathrm{LD}}$ is predominantly used for the identification of future bull dams but the main part of $\Delta \mathrm{G}_{\mathrm{a}}$ is still generated from selection of sires. The low selection intensity in the path dam to sire induced a higher sensitivity of $\Delta \mathrm{G}_{\mathrm{a}}$ to changes in cost and accuracies of GEBV $\mathrm{LD}_{\mathrm{LD}}$ compared with the same changes of $\mathrm{GEBV}_{\mathrm{HD}}$. On the contrary, the genetic gain generated from selection of males was only affected by changes in accuracies of GEBV $_{\mathrm{HD}}$ but almost unaffected by any changes in cost. Thus, changes in cost and accuracies of $\mathrm{GEBV}_{\mathrm{LD}}$ put the most pressure on the breeding scheme structure to maintain a high $\Delta \mathrm{G}_{\mathrm{a}}$. Furthermore, genomic selection of bull dams produced by far the majority of breeding cost but the lowest genetic gain.
\end{abstract}

Key words: multistage selection, genomic selection, dairy cattle

Received March 22, 2011.

Accepted November 14, 2011.

${ }^{1}$ Corresponding author: reinsch@fbn-dummerstorf.de

\section{INTRODUCTION}

Genomic selection (GS) of farm animals is revolutionizing animal breeding in theory and practice (Meuwissen et al., 2001). Highly dense maps of SNP markers in linkage disequilibrium with QTL coding for economically important traits allow the derivation of genomic EBV (GEBV). Selecting individuals on the basis of GEBV tackles 3 major frontiers of animal breeding: the accuracy of breeding values for traits with a low heritability (Calus and Veerkamp, 2007; Muir, 2007), the control of inbreeding (Daetwyler et al., 2007), and the generation interval (L; Schaeffer, 2006). Because L is the major obstacle for increasing the genetic gain per year $\left(\Delta \mathbf{G}_{\mathbf{a}}\right)$ in dairy cattle breeding, it has received the most attention from researchers. Although accuracies of GEBV ( $\mathbf{r}_{\text {GEBV }}$ ) for milk production are still below those of conventional progeny-testing schemes (Hayes et al., 2009), an increase in $\Delta \mathrm{G}_{\mathrm{a}}$ can be achieved with a decreased L (Schaeffer, 2006). In a detailed calculation, Schaeffer (2006) summarized the potential effects of GS on dairy cattle breeding schemes assuming a 1-stage selection approach and highly accurate GEBV available for male selection candidates and all potential bull dams. The latter might not be realistic, as genotyping large proportions of the cow population may be impossible due to financial limitations. Furthermore, if overall breeding costs are limited and costs for GEBV $\left(\mathbf{C}_{\mathrm{GEBV}}\right)$ are high, the small surplus in genetic gain from GS of bull dams due to low selection intensity might not be justifiable.

As SNP chips of different densities are available, financial limitations may be overcome by using cheap low-density chips where SNP are subsets of high-density chips and evenly spaced across the genome. Imputation algorithms can be applied to derive high-density genotypes from low-density genotypes where the loss in accuracy of GEBV estimated from imputed genotypes is reported to be between 0 and $45 \%$, depending on the construction of the subset (Habier et al., 2009; VanRaden, 2010; Weigel et al., 2010).

As a consequence, the decision-making processes required by breeding organizations concerning the detailed structure of breeding schemes have become very complex. This complexity is based first on a possible 
change in the realized $\mathrm{r}_{\mathrm{GEBV}}$ of breeding values from high- and low-density SNP genotypes due to technological improvements, accompanied by changes in future genotyping costs. Second, GS and the availability of different SNP chips allow for diverse kinds of single-, 2-, or multistage selection in every selection path, whereas economic resources are not selection path specific and limited. Decisions on the selection design in one path affect accuracies, selection intensities, and $\mathrm{L}$ in other paths. Thus, answers to questions concerning whether, where, when, and at what cost certain information correlated with the aggregate genotype should be gathered are important for the breeding program as a whole. Moreover, decisions must be made despite the uncertainty of future developments of important parameters such as $\mathrm{r}_{\mathrm{GEBV}}$ and $\mathrm{C}_{\mathrm{GEBV}}$.

The aim of this study was to investigate the variability of $\Delta \mathrm{G}_{\mathrm{a}}$ and the effect of changes in $\mathrm{r}_{\mathrm{GEBV}}$ and $\mathrm{C}_{\mathrm{GEBV}}$ on optimized dairy cattle schemes when GS is practiced on the basis of a high-density $\left(\mathbf{G S}_{\mathbf{H D}}\right)$ and a lowdensity $\left(\mathbf{G S}_{\mathbf{L D}}\right)$ SNP chip with financial constraints. The costs $\left(\mathbf{C}_{\mathrm{GEBV}, \mathrm{HD}}\right)$ and accuracies $\left(\mathbf{r}_{\mathrm{GEBV}, \mathrm{HD}}\right)$ of breeding values $\left(\mathbf{G E B V}_{\mathbf{H D}}\right)$ estimated from a highdensity and a low-density SNP chip (with respective parameters $\mathbf{C}_{\mathbf{G E B V , L D}}, \mathbf{r}_{\mathrm{GEBV}, \mathrm{LD}}$, and $\left.\mathbf{G E B V} \mathbf{V}_{\mathbf{L D}}\right)$ were varied in a semi-continuous manner. Optimum breeding schemes for each combination of $\mathrm{C}_{\mathrm{GEBV}}$ and $\mathrm{r}_{\mathrm{GEBV}}$ were identified using a grid search varying the proportion of animals selected at all possible stages of every selection path. The results provide insights into the dynamics of resource allocation among selection paths as well as identifying the main sources of $\Delta \mathrm{G}_{\mathrm{a}}$ and the sensitivity of $\Delta \mathrm{G}_{\mathrm{a}}$ and breeding scheme structures to changes in $\mathrm{r}_{\mathrm{GEBV}}$ and $\mathrm{C}_{\mathrm{GEBV}}$.

\section{METHODS}

Deterministic methods were used for optimizing breeding plans. Accuracies of predicted breeding values were derived from selection index theory; selection intensities after $\mathrm{n}$ selection stages were obtained via numerical integration of a truncated multivariate normal distribution. The pedigree and associated phenotypic and genomic information for candidates and relatives available for constructing case-specific selection indices are given in Figure 1.

\section{Consideration of GEBV}

Following the approach of Dekkers (2007), GEBV $\mathrm{HD}_{\mathrm{D}}$ and $\mathrm{GEBV}_{\mathrm{LD}}$ were included in the selection index as traits with heritabilities of 1 and genetic correlations determined by their accuracies. The correlation between $\mathrm{GEBV}_{\mathrm{HD}}$ and $\mathrm{GEBV}_{\mathrm{LD}}$ was calculated:

$$
r_{\mathrm{HD}, \mathrm{LD}}=\frac{\sigma_{\mathrm{LD}}}{\sigma_{\mathrm{HD}}},
$$

where $r_{\mathrm{HD}, \mathrm{LD}}$ is the correlation between $\mathrm{GEBV}_{\mathrm{HD}}$ and $\mathrm{GEBV}_{\mathrm{LD}}, \sigma_{\mathrm{LD}}$ is the standard deviation of $\mathrm{GEBV}_{\mathrm{LD}}$, and $\sigma_{\mathrm{HD}}$ is the standard deviation of $\mathrm{GEBV}_{\mathrm{HD}}$. A detailed derivation of this formula is given in the Appendix.

\section{Genetic Gain}

The genetic gain $(\Delta G)$ per generation of selection path $i\left(\Delta G_{i}\right)$ was calculated according to

$$
\Delta G_{i}=z_{i, n} \sigma_{a}
$$

where $z_{i, n}$ is the selection intensity on the aggregate genotype after selecting at the last of $j=1, \ldots, n$ stages and $\sigma_{a}$ is the standard deviation of the aggregate genotype. It was assumed that both aggregate genotype and selection indices are multivariate normal distributed (Cochran, 1951). Therefore, the selection intensity was determined using the moment generating function of a truncated multivariate normal distribution (Tallis, 1961) where the truncation points were calculated following Mi and Utz (2008) and combining integration algorithms of Genz (1992) and maximization techniques of Brent (1973).

The $\Delta G_{a}$ for the whole breeding scheme was calculated following Rendel and Robertson (1950):

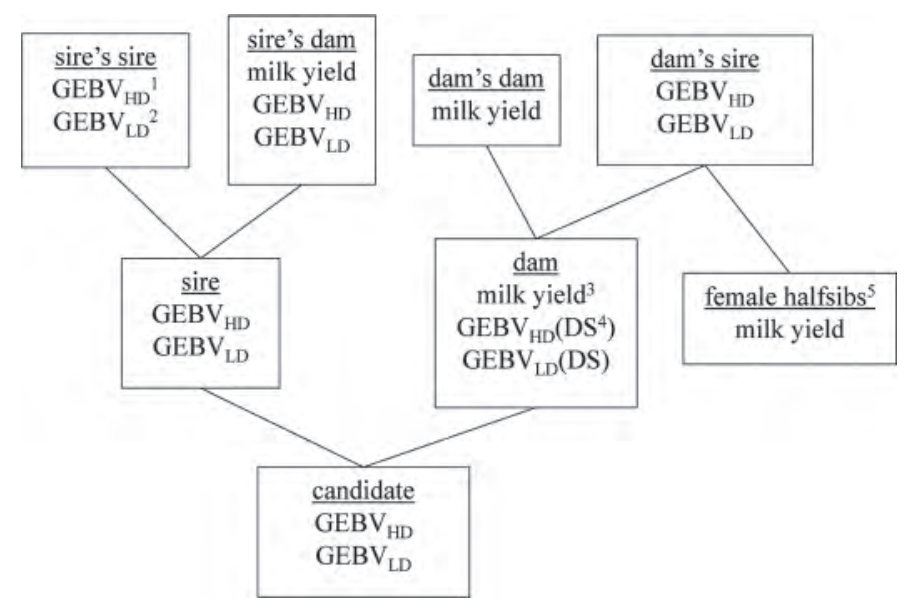

Figure 1. Standard pedigree used to derive breeding values in all selection paths. ${ }^{1}$ Accuracy of breeding values estimated from high-density SNP marker genotypes. ${ }^{2}$ Accuracy of breeding values estimated from low-density SNP marker genotypes. ${ }^{3}$ The trait is only available if this ancestor was selected from the path dam-dam and the candidate is selected from the path dam-sire. ${ }^{4}$ The trait is only available if the ancestor was selected from the path dam-sire and the candidate is selected from the path sire-sire or sire-dam. ${ }^{5}$ The informant and trait is only available if the dam was selected from the path dam-dam and the candidate is selected from the path dam-sire. 


$$
\Delta G_{a}=\frac{\sum_{i} \Delta G_{i}}{\sum_{i} L_{i}},
$$

where $L_{i}$ is the generation interval in path $i$.

\section{Breeding Program}

A dairy cattle breeding program with a cow population of 100,000 was modeled. Bull dams were selected from all available heifers and assumed to be used as bull dams only once. Male selection candidates were produced by contract matings to bull dams. For the sake of simplicity, there was only 1 milk trait with a heritability of 0.25 in the breeding goal. Selection could take place on based on pedigree data, $\mathrm{GEBV}_{\mathrm{LD}}$, and $\mathrm{GEBV}_{\mathrm{HD}}$.

Details on biological, technical, and economic parameters are given in Table 1. All parameters expressed in terms of probabilities and not given in Table 1 are assumed to be 0 or 1 (e.g., success of first insemination, survival rate of calves, among others).

Conventional selection paths are sire to sire (SS), sire to dam (SDm), dam to sire (DS), and dam to dam (DD). As almost no selection takes place in the path DD, a selection intensity of 0 was assumed for this path. Each selection path was structured in stages. Each stage was characterized by the amount of information available on the selection candidates. The selection stages in the paths SS, SDm, and DS were a) selection on performance data and available GEBV of ancestors and half sibs (hereafter termed pedigree stage), b) selection on the candidate's $\mathrm{GEBV}_{\mathrm{LD}}\left(\mathrm{GS}_{\mathrm{LD}}\right.$ stage), and c) selection on the candidate's $\mathrm{GEBV}_{\mathrm{HD}}\left(\mathrm{GS}_{\mathrm{HD}}\right.$ stage).

For GS of male calves a tissue sample was taken by a veterinarian on farm at birth and was paid for by the farmer. The DNA isolation and SNP genotyping were carried out by a central laboratory at the expense of the breeding organization. Selection candidates were kept on farm until the age of 6 mo. Farmers keeping male candidates selected at the pedigree stage and slaughtered after the first (low-density) or second (highdensity) genotyping were financially compensated by the breeding organization to cover the costs of keeping the candidates during each genotyping process. In the case of genotyping female calves as potential bull dams the division of the costs was the same, but no compensation was paid because females were not acquired by the breeding organization.

Table 1. Biological, economic, and technical parameters of the breeding program

\begin{tabular}{|c|c|c|}
\hline Parameter & Unit & Value \\
\hline $\begin{array}{l}\text { Heritability }\left(\mathrm{h}^{2}\right) \text { of milk trait } \\
\text { Phenotypic SD } \\
\mathrm{r}_{\mathrm{GEBV}, \mathrm{HD}_{1}} \\
\mathrm{r}_{\mathrm{GEBV}, \mathrm{LD}} \\
\text { Age at first calving } \\
\text { Calving interval } \\
\text { Length of lactation } \\
\text { Maturity of test bulls } \\
\text { Average age of bull calves at purchase } \\
\text { Generation interval of bull/cow sires } \\
\text { Generation interval of bull dams } \\
\text { Generation interval of cow dams } \\
\text { Price of bull calves } \\
\text { Husbandry cost of bull calves until maturity } \\
\mathrm{C}_{\mathrm{GEBV}, \mathrm{HD}}, 4 \\
\mathrm{C}_{\mathrm{GEBV}, \mathrm{LD}} \\
\text { Population of cows } \\
\text { Demand for cow sires } \\
\text { Demand for bull sires } \\
\text { Initial male selection candidates } \\
\text { Demand for bull dams (contract matings) } \\
\text { Compensation payments for husbanding male calves until } \mathrm{GEBV}_{\mathrm{LD}}{ }^{6} \text { estimation } \\
\text { Compensation payments for husbanding male calves until } \mathrm{GEBV}_{\mathrm{HD}}{ }^{2} \text { estimation } \\
\text { Maximum breeding cost }\end{array}$ & $\begin{array}{l}\text { mo } \\
\text { mo } \\
\text { mo } \\
\text { mo } \\
\text { mo } \\
\text { mo } \\
\text { mo } \\
\text { mo } \\
€ \\
€ / d \\
€ \\
€ \\
\text { Animals } \\
\text { Animals /yr } \\
\text { Animals /yr } \\
\text { Animals /yr } \\
\text { Animals /yr } \\
€ / \text { calf } \\
€ / \text { calf } \\
€ / y r\end{array}$ & $\begin{array}{c}0.25 \\
700 \\
0.7-0.9 \\
0.4-0.65 \\
26 \\
12 \\
10 \\
14 \\
6 \\
23 \\
26 \\
48 \\
4,000 \\
5 \\
150-250 \\
20-100 \\
100,000 \\
10 \\
5 \\
500 \\
1,000 \\
150 \\
150 \\
719,050\end{array}$ \\
\hline
\end{tabular}

${ }^{1}$ Accuracy of breeding values estimated from high-density SNP marker genotypes.

${ }^{2}$ Accuracy of breeding values estimated from low-density SNP marker genotypes.

${ }^{3}$ Cost of breeding values estimated from high-density SNP marker genotypes per genotyped individual.

${ }^{4}$ Costs include DNA isolation, genotyping, and calculation of breeding values but no blood sample recovery.

${ }^{5}$ Cost of breeding values estimated from low-density SNP marker genotypes per genotyped individual.

${ }^{6}$ Breeding values estimated from high-density SNP marker genotypes.

${ }^{7}$ Breeding values estimated from low-density SNP marker genotypes. 


\section{Breeding Cost}

Costs independent of selection strategies (e.g., performance recording of females, calculating pedigree index, marketing, and semen processing) were not considered. To allow for reduced costs for labor and infrastructure incurred by different selection schemes, all expenses were derived from some invariant basic costs per animal using the cost function of Namkoong (1970):

$$
C_{i b}=C_{b} n_{i} \Pi_{j=1}^{k-1} p_{i j},
$$

where $C_{i b}$ are the costs of expense factor $b$ in path $i, C_{b}$ are the costs per individual of this expense factor, $n_{i}$ is the number of initial selection candidates, $p_{i j}$ is the probability that a candidate selected at stage $j$ moves on to stage $j+1$, and $k$ is the stage where the costs occur; $C_{b}$ can be purchasing, genotyping, or trait measurement costs, or compensation payments. In the case of compensation payments, the formula changes to

$$
C_{i b}=C_{b} n_{i} \Pi_{j=1}^{k-1} p_{i j}\left(1-p_{i k}\right) .
$$

The total cost in path $i$ is the sum over all expense factors in the path, and the total breeding cost is the sum over all paths.

The maximum breeding cost of $719.050 €$ was imposed as a constraint during maximization and was derived assuming progeny performance testing of 50 young bulls per year:

$$
\begin{gathered}
(4,000 €+213.5 \mathrm{~d} \times 5 € / \mathrm{d}+1,403 \mathrm{~d} \\
\times 4.5 € / \mathrm{d}+3,000 €) \times 50 .
\end{gathered}
$$

This cost included purchasing 50 male calves from contract matings, keeping them until maturity, maintenance from maturity until breeding value estimation from daughter records, and compensation payments for test bull insemination.

\section{Parameter Variation}

Costs and accuracies of GEBV were varied in the following manner: $\mathrm{r}_{\mathrm{GEBV}, \mathrm{HD}}$ between 0.7 and 0.9 and $\mathrm{r}_{\mathrm{GEBV}, \mathrm{LD}}$ between 0.4 and 0.65 , both in steps of 0.05 ; $\mathrm{C}_{\mathrm{GEBV}, \mathrm{HD}}$ between $150 €$ and $250 €$ and $\mathrm{C}_{\mathrm{GEBV}, \mathrm{LD}}$ between $20 €$ and $100 €$, both in steps of $20 €$, resulting in 900 combinations by cross-classifying all 4 parameters.

\section{Maximization}

Breeding schemes maximizing $\Delta \mathrm{G}_{\mathrm{a}}$ for each combination of $\mathrm{C}_{\mathrm{GEBV}, \mathrm{HD}}, \mathrm{C}_{\mathrm{GEBV}, \mathrm{LD}}, \mathrm{r}_{\mathrm{GEBV}, \mathrm{HD}}$, and $\mathrm{r}_{\mathrm{GEBV}, \mathrm{LD}}$ were obtained using a grid search varying the proportion of selected individuals at each selection stage in every path. Trait measurements were available for ancestors within the maximization process and, therefore, the accuracy of the selection index was adjusted according to the selection strategy in the path from which the ancestor had been derived. The possibility of an additional selection stage was allowed for selecting bull sires from cow sires, requiring additional information (e.g., $\mathrm{GEBV}_{\mathrm{HD}}$ ) instead of just increased selection intensity for this group of males.

For each path, the initial number of selection candidates and the final number of selected individuals for reproduction were fixed. The product of $p_{i j}$ values had to fulfill the equation

$$
\frac{s_{i}}{n_{i}}=\prod_{j=1}^{m_{i}} p_{i j}
$$

where $s_{i}$ is the number of selected individuals actually used for reproduction and $m_{i}$ is the number of selection stages in path $i$.

The proportion $p_{i j}$ was varied between 0.01 and 1 in steps of 0.05 . Stages with $p_{i j}=1$ were treated as if the selection stage was skipped. For the used stages $\left(p_{i j}<1\right)$ the constraint of Equation 7 was fulfilled by calculating $p_{i j}$ of the last used stage as a dependent variable. The stage combination was accepted as valid only if this value was $<1$. For each combination of parameters, the valid stage combinations of all paths were completely cross-classified to obtain all possible breeding schemes. Breeding costs were derived for each of these schemes but $\Delta \mathrm{G}_{\mathrm{a}}$ was only calculated if the cost constraint was fulfilled. The breeding scheme with the highest $\Delta G_{a}$ was seen as the optimum for the given combination of $\mathrm{C}_{\mathrm{GEBV}}$ and $\mathrm{r}_{\mathrm{GEBV}}$.

To compare the extent of $\mathrm{GS}_{\mathrm{LD}}$ and $\mathrm{GS}_{\mathrm{HD}}$ between breeding schemes, the proportion of initial selection candidates high-density $\left(\mathbf{P G}_{\mathbf{H D}}\right)$ and low-density $\left(\mathbf{P G}_{\mathbf{L D}}\right)$ genotyped in each path $\left(P G_{H D, i}, P G_{L D, i}\right)$ was calculated according to

$$
\begin{aligned}
P G_{H D, i} & =\prod_{j=1}^{k_{H D}-1} p_{i j} \\
P G_{L D, i} & =\prod_{j=1}^{k_{L D}-1} p_{i j},
\end{aligned}
$$

where $k_{H D}$ is the $\mathrm{GS}_{\mathrm{HD}}$ stage and $k_{L D}$ is the $\mathrm{GS}_{\mathrm{LD}}$ stage within selection path $i$.

Across all 900 possible combinations of input parameters, the total number of evaluated breeding plans in terms of overall breeding cost was 146 million (20 possible $p_{i j}$ values at each selection stage, $20^{3}$ possible combinations of stage selection intensities in each path, and 
$20^{9}$ possible breeding schemes from cross-classification of 3 paths, excluding those schemes where the selection probabilities in at least 1 path did not fulfill Equation 7); 6.7 million of these plans fulfilled the cost constraint and were compared concerning $\Delta \mathrm{G}_{\mathrm{a}}$.

\section{Breeding Scheme Similarity Indicator}

To analyze the effect of the variation of $\mathrm{C}_{\mathrm{GEBV}}$ and $\mathrm{r}_{\mathrm{GEBV}}$ on the structure of breeding schemes in terms of changes of $\mathrm{PG}_{\mathrm{HD}}$ and $\mathrm{PG}_{\mathrm{LD}}$, a correlation-like similarity measurement was constructed as follows: let $\mathbf{x}_{i, 1}$ be a vector of the selection path $i$ within breeding scheme $l$ with a dimension of the possible (but not necessarily used) selection stages within that path, excluding the pedigree stage. The values within $\mathbf{x}_{i, 1}$ are $\mathrm{PG}_{\mathrm{HD}}$ and $\mathrm{PG}_{\mathrm{LD}}$ in path $i$ of breeding scheme $l$, where these values were set to 0 if $\mathrm{GS}_{\mathrm{HD}}$ or $\mathrm{GS}_{\mathrm{LD}}$, or both, were not applied in that path. Furthermore, let $\mathbf{x}_{1}=\left(\mathbf{x}_{\mathrm{SS}, 1}, \mathbf{x}_{\mathrm{SD}, 1}, \mathbf{x}_{\mathrm{DS}, 1}\right)$ be a vector from concatenating all $\mathbf{x}_{i, 1}$ vectors of the breeding scheme $l$, and $\overline{\mathbf{x}}_{l}$ the mean of $\mathbf{x}_{l}$. With the similarity between the structure of the breeding schemes $l$ and $g$, $s_{l g}$ was then

$$
s_{l g}=\frac{\frac{\mathbf{x}_{l}^{\prime} \mathbf{x}_{g}}{n}-\bar{x}_{l} \bar{x}_{g}}{\sqrt{\left(\frac{\mathbf{x}_{l}^{\prime} \mathbf{x}_{l}}{n}-\bar{x}_{l}^{2}\right)\left(\frac{\mathbf{x}_{\mathrm{g}}^{\prime} \mathbf{x}_{g}}{n}-\bar{x}_{\mathrm{g}}^{2}\right)}},
$$

where $n$ is the dimension of $\mathbf{x}_{1}$ and $\mathbf{x}_{\mathrm{g}}$. The possible values of $s_{l q}$ range from -1 to 1 , where a value of 1 indicates identification of 2 identical breeding schemes with regard to the proportion selected at each possible selection stage.

\section{Software}

All calculations were carried out with a FORTRAN 90 program written by the first author. The FORTRAN routines of Genz (1992) and Brent (1973) were used to calculate the selection intensity in a multistage breeding program.

\section{RESULTS}

\section{Optimization Results}

Some properties were shared by all breeding schemes maximizing $\Delta \mathrm{G}_{\mathrm{a}}$. No additional selection stage was introduced for selecting bull sires from the group of cow sires. Instead, both groups of males were always selected on the same amount of information, but with different selection intensities. In particular, no breeding scheme where cow sires were selected on $\mathrm{GEBV}_{\mathrm{LD}}$ and bull sires on $\mathrm{GEBV}_{\mathrm{HD}}$ in the last stage was ever observed as optimum. Moreover, bulls were never selected only on pedigree information or pedigree plus $\mathrm{GEBV}_{\mathrm{LD}}$. Likewise, bull dams were never selected solely on pedigree information alone, but always on a combination of pedigree with $\mathrm{GS}_{\mathrm{LD}}$ or $\mathrm{GS}_{\mathrm{HD}}$. The largest proportion of breeding cost was always produced by GS of bull dams. Furthermore, in all cases the contributions of the different selection paths to $\sum_{\mathrm{i}} \Delta \mathrm{G}_{\mathrm{i}}$ were in the following order: $\Delta \mathrm{G}_{\mathrm{SS}}>\Delta \mathrm{G}_{\mathrm{SDm}}>\Delta \mathrm{G}_{\mathrm{DS}}$.

Table 2 summarizes the results of the $\Delta \mathrm{G}_{\mathrm{a}}, \Delta \mathrm{G}_{\mathrm{SS}}$, $\Delta \mathrm{G}_{\mathrm{SDm}}, \Delta \mathrm{G}_{\mathrm{DS}}$, absolute and relative total breeding costs, $\mathrm{PG}_{\mathrm{HD}, \mathrm{SDm}}, \mathrm{PG}_{\mathrm{LD}, \mathrm{SDm}}, \mathrm{PG}_{\mathrm{HD}, \mathrm{DS}}$, and $\mathrm{PG}_{\mathrm{LD}, \mathrm{DS}}$ in terms of mean, maximum, and minimum across all input parameter combinations. The results for a particular scenario with a given parameter combination of $\mathrm{r}_{\mathrm{GEBV}, \mathrm{HD}}=0.75, \mathrm{r}_{\mathrm{GEBV}, \mathrm{LD}}=0.6, \mathrm{C}_{\mathrm{GEBV}, \mathrm{HD}}=210 €$, and $\mathrm{C}_{\mathrm{GEBV}, \mathrm{LD}}=100 €$ are given as a reference in the last column of Table 2. The $\Delta \mathrm{G}_{\mathrm{a}}$ varied between 223 and $161 \mathrm{~kg}$ of milk, corresponding to 0.64 and 0.46 genetic standard deviations, respectively. Overall genetic gain across all pathways was mainly generated due to the selection of sires, whereas the contribution of bull dams never exceeded $31 \%$. A $\mathrm{PG}_{\mathrm{HD}, \mathrm{SDm}}$ range between 1 and 0.06 indicates that $\mathrm{GS}_{\mathrm{HD}}$ was always applied to select cow sires, whereas the magnitude of $\mathrm{GS}_{\mathrm{HD}}$ in the path DS never exceeded 0.0265 , and parameter combinations exist excluding $\mathrm{GS}_{\mathrm{HD}}$ from this path. In some parameter combinations (e.g., the reference scenario), the breeding schemes maximizing $\Delta \mathrm{G}_{\mathrm{a}}$ were characterized by a $P G_{L D, S D m}$ value of 0.95 . Because selection of males only on pedigree information and $\mathrm{GEBV}_{\mathrm{LD}}$ was never found in optimum breeding schemes, such a $\mathrm{PG}_{\mathrm{LD}, \mathrm{SDm}}$ value implied genotyping $95 \%$ of the male selection candidates, followed by a further selection on GEB$\mathrm{V}_{\mathrm{HD}}$. Dependent on genotyping cost, up to $55 \%$ of the entire heifer population was proposed to be low-density genotyped, but huge differences between $\mathrm{r}_{\mathrm{GEBV}, \mathrm{HD}}$ and $\mathrm{r}_{\mathrm{GEBV}, \mathrm{LD}}$ led to $\mathrm{GS}_{\mathrm{HD}}$ selection of only a trivial number of heifers instead (results not shown). Dictated by the number of initial selection candidates and the cost restriction, the means of $\mathrm{PG}_{\mathrm{HD}, \mathrm{DS}}$ and $\mathrm{PG}_{\mathrm{LD}, \mathrm{DS}}$ were much lower compared than those of the path SDm.

The complex interaction between input parameters is mirrored by the multiple regression coefficients of various results on the relative input parameters given in Table 3 (in the regression analysis, input parameters were expressed as proportions of their respective maxima). The highest positive effect on $\Delta \mathrm{G}_{\mathrm{a}}$ of all input parameters can be attributed to $\mathrm{r}_{\mathrm{GEBV}, \mathrm{HD}}$, which was 9 times higher than that of $\mathrm{r}_{\mathrm{GEBV}, \mathrm{LD}}$. Increasing $\mathrm{C}_{\mathrm{GEBV}}$ should negatively affect $\Delta \mathrm{G}_{\mathrm{a}}$, but interestingly, 
Table 2. Results for parameters across accuracies and cost of genomic estimated breeding values (\% in parentheses)

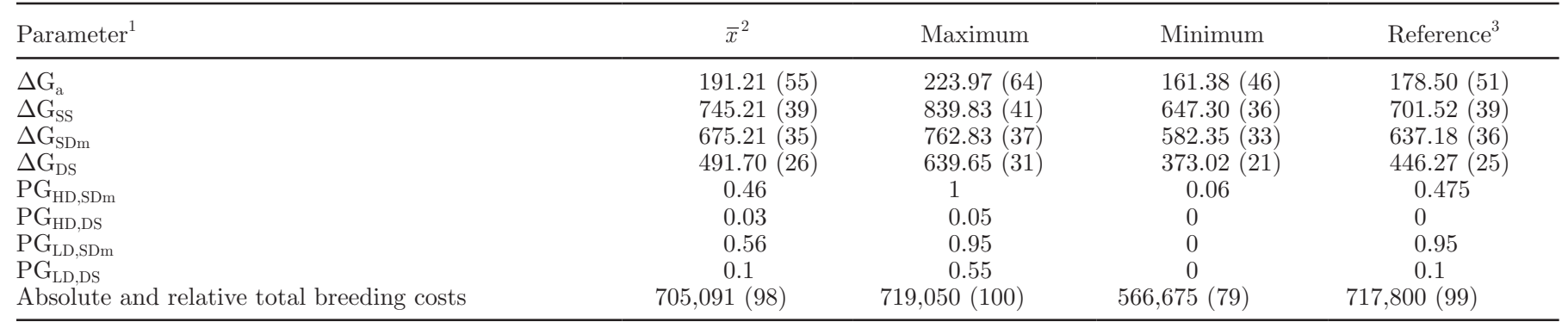

${ }^{1} \Delta \mathrm{G}_{\mathrm{a}}=$ genetic gain per year and as a proportion of the additive genetic variance; $\Delta \mathrm{G}_{\mathrm{SS}}=$ genetic gain per generation in the path sire-sire and as a proportion of the total genetic gain; $\Delta \mathrm{G}_{\mathrm{SDm}}=$ genetic gain per generation in the path sire-dam and as a proportion of the total genetic gain; $\Delta \mathrm{G}_{\mathrm{DS}}=$ genetic gain per generation in the path dam-sire and as a proportion of the total genetic gain; $\mathrm{PG}_{\mathrm{HD}, \mathrm{SDm}}=$ proportion of the initial selection candidates in the path sire-dam being genotyped with a high-density $\mathrm{SNP}$ chip; $\mathrm{PG}_{\mathrm{HD}, \mathrm{DS}}=$ proportion of the initial selection candidates in the path dam-sire being genotyped with a high-density SNP chip; $\mathrm{PG}_{\mathrm{LD} . \mathrm{SDm}}=$ proportion of the initial selection candidates in the path sire-dam being genotyped with a low-density SNP chip; $\mathrm{PG}_{\mathrm{LD}, \mathrm{DS}}=$ proportion of the initial selection candidates in the path dam-sire being genotyped with a low-density SNP chip.

${ }^{2}$ The mean.

${ }^{3}$ Calculation results for a parameter combination of $\mathrm{r}_{\mathrm{GEBV}, \mathrm{HD}}=0.75, \mathrm{r}_{\mathrm{GEBV}, \mathrm{LD}}=0.6, \mathrm{C}_{\mathrm{GEBV}, \mathrm{HD}}=210 €$, and $\mathrm{C}_{\mathrm{GEBV}, \mathrm{LD}}=100 € . \mathrm{r}_{\mathrm{GEBV}, \mathrm{HD}}$ and $\mathrm{r}_{\mathrm{GEBV}, \mathrm{LD}}$ $=$ the accuracy of breeding values estimated from a high-density or low-density SNP chip, respectively. $\mathrm{C}_{\mathrm{GEBV}-\mathrm{HD}}$ and $\mathrm{C}_{\mathrm{GEBV}-\mathrm{LD}}=$ the costs of breeding values estimated from a high-density or low-density SNP chip, respectively.

the effect of $\mathrm{C}_{\mathrm{GEBV}, \mathrm{LD}}$ was 3 times higher than that of $\mathrm{C}_{\mathrm{GEBV}, \mathrm{HD}}$. Furthermore, an increase in $\mathrm{C}_{\mathrm{GEBV}, \mathrm{LD}}$ affected $\Delta \mathrm{G}_{\mathrm{SDm}}$ positively and a higher value for $\mathrm{r}_{\mathrm{GEBV} . \mathrm{LD}}$ had only a very small effect on $\Delta \mathrm{G}_{\mathrm{SDm}}$. The effect of all varied parameters on genotyping males was at least as twice as high as on genotyping females. In particular, $\mathrm{C}_{\text {GEBV LDD }}$ had almost no effect on $\mathrm{PG}_{\mathrm{HD}, \mathrm{DS}}$, whereas its effect on $\mathrm{PG}_{\mathrm{HD}, \mathrm{SDm}}$ was almost 850 times higher, although $\Delta \mathrm{G}_{\mathrm{a}}$ was mainly generated in the path SDm and SS, respectively, due to $\mathrm{GS}_{\mathrm{HD}}$.

The results of Tables 2 and 3 show the nonlinearity of the genetic gain as a function of the proportion of genotyped individuals and, therefore, of the selection intensity. It must also be kept in mind that a decrease in the proportion of genotyped dams of $5 \%$ (which is equal to 2,500 animals) allowed almost all male selection candidates to be genotyped. Figure 2 reflects $\Delta G_{a}$ of the reference scheme as a function of $P G_{L D, S D m}$ $\left(\mathrm{PG}_{\mathrm{HD}, \mathrm{SDm}}, \mathrm{PG}_{\mathrm{LD}, \mathrm{DS}}\right.$, and $\mathrm{PG}_{\mathrm{HD}, \mathrm{DS}}$ were chosen such that $\Delta G_{\mathrm{a}}$ was maximized) and as a function of $\mathrm{PG}_{\mathrm{HD}, \mathrm{SDm}}$ for a given $\mathrm{PG}_{\mathrm{LD}, \mathrm{SDm}}\left(\mathrm{PG}\right.$ LD,DS$\left.=0.05, \mathrm{PG}_{\mathrm{HD}, \mathrm{DS}}=0\right)$. Note that in Figure $2 \mathrm{a}$, a $\mathrm{PG}_{\mathrm{LD}, \mathrm{SDm}}$ of 0 indicates a selection of males only on pedigree index and $\mathrm{GEBV}_{\mathrm{HD}}$. A high or even $0 \mathrm{PG}_{\mathrm{LD}, \mathrm{SDm}}$ allowed $\mathrm{PG}_{\mathrm{HD}, \mathrm{SDm}}$ to vary in a wider range, resulting in numerous values for $\mathrm{PG}_{\mathrm{HD}, \mathrm{SDm}}$ yielding almost the same $\Delta \mathrm{G}_{\mathrm{a}}$. Thus, $\mathrm{PG}_{\mathrm{LD}, \mathrm{SDm}}$ and

Table 3. Regression coefficients of the genetic gain and proportion of genotyped individuals in different selection paths on varied input parameters ${ }^{1}$

\begin{tabular}{lcccc}
\hline Parameter $^{2}$ & $\mathrm{r}_{\mathrm{GEBV}, \mathrm{HD}}{ }^{3}$ & $\mathrm{r}_{\mathrm{GEBV}, \mathrm{LD}}{ }^{4}$ & $\mathrm{C}_{\mathrm{GEBV}, \mathrm{HD}}{ }^{5}$ & $\mathrm{C}_{\mathrm{GEBV}, \mathrm{LD}}{ }^{6}$ \\
\hline$\Delta \mathrm{G}_{\mathrm{a}}$ & 204 & 23 & -3.19 & -10 \\
$\Delta \mathrm{G}_{\mathrm{SDm}}$ & 753 & 9 & -3.66 & 1.08 \\
$\Delta \mathrm{G}_{\mathrm{DS}}$ & 455 & 212 & -26 & -108 \\
$\mathrm{PG}_{\mathrm{HD}, \mathrm{SDm}}$ & 0.83 & -0.46 & -0.24 & 0.48 \\
$\mathrm{PG}_{\mathrm{LD}, \mathrm{SDm}}$ & -0.58 & 1.29 & 0.37 & -0.45 \\
$\mathrm{PG} \mathrm{G}_{\mathrm{HD}, \mathrm{DS}}$ & 0.11 & -0.05 & -0.02 & 0.00057 \\
$\mathrm{PG}_{\mathrm{LD}, \mathrm{DS}}$ & -0.07 & 0.31 & 0.04 & -0.21 \\
\hline
\end{tabular}

${ }^{1}$ In the regression analysis, input parameters were expressed as a proportion of the respective maximum.

${ }^{2} \Delta \mathrm{G}_{\mathrm{a}}=$ genetic gain per year; $\Delta \mathrm{G}_{\mathrm{SDm}}=$ genetic gain per generation in the path sire-dam; $\Delta \mathrm{G}_{\mathrm{DS}}=$ genetic gain per generation in the path dam-sire; $\mathrm{PG}_{\mathrm{HD} \text { SDm }}=$ proportion of the initial selection candidates in the path siredam being genotyped with a high-density SNP chip; $\mathrm{PG}_{\mathrm{LD}, \mathrm{SDm}}=$ proportion of the initial selection candidates in the path sire-dam being genotyped with a low-density SNP chip; $\mathrm{PG}_{\mathrm{HD} \text {.DS }}=$ proportion of the initial selection candidates in the path dam-sire being genotyped with a high-density $\mathrm{SNP}$ chip; $\mathrm{PG}_{\mathrm{LD}, \mathrm{DS}}=$ proportion of the initial selection candidates in the path dam-sire being genotyped with a low-density SNP chip.

${ }^{3}$ The accuracy of breeding values estimated from a high-density SNP chip.

${ }^{4}$ The accuracy of breeding values estimated from a low-density SNP chip.

${ }^{5}$ The costs of breeding values estimated from a high-density SNP chip.

${ }^{6}$ The costs of breeding values estimated from a low-density SNP chip. 


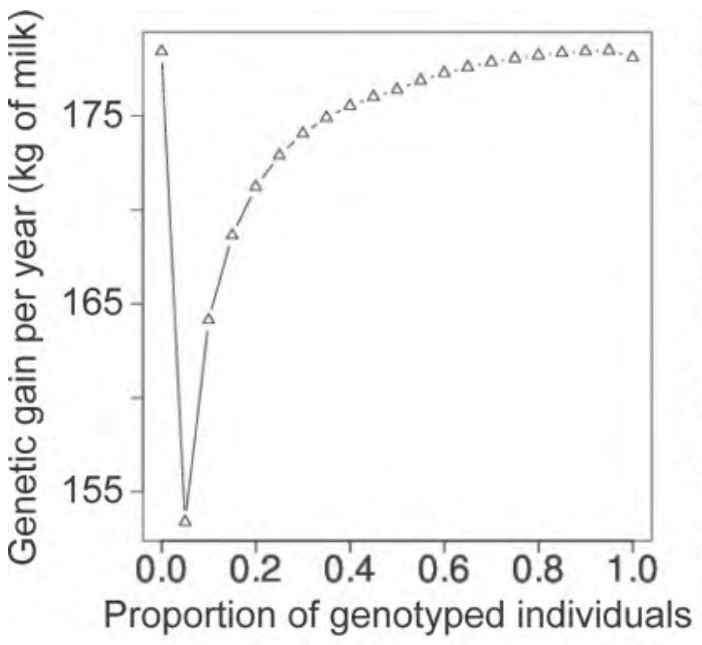

(a)

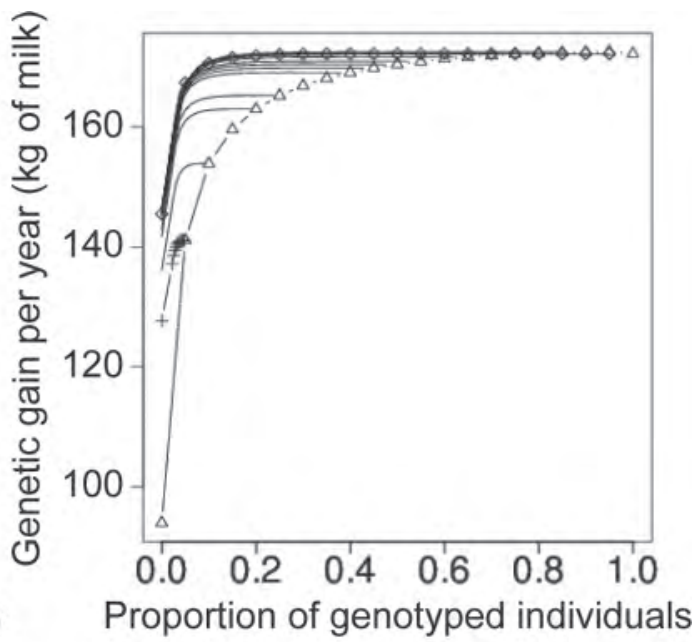

(b)

Figure 2. Genetic gain per year $\left(\Delta \mathrm{G}_{\mathrm{a}}\right.$, $\mathrm{kg}$ of milk) as a function of low- (a) and high- (b) density genotyped male selection candidates. Underlying costs and accuracies of genomically estimated breeding values are equal to the reference scheme. Figure $2 \mathrm{a}$ mirrors $\Delta \mathrm{G}_{\mathrm{a}}$ if the proportion of high-density genotyped male selection candidates in the path sire to dam $\left(\mathrm{PG}_{\mathrm{HD} \text { SDm }}\right)$ and all other variables are chosen such that $\Delta \mathrm{G}_{\mathrm{a}}$ is maximized. In Figure $2 \mathrm{~b}$, the $\mathrm{PG}_{\mathrm{HD}, \mathrm{SDm}}$ is varied for a given proportion of low-density genotyped male selection candidates in the path sire to dam $\left(\mathrm{PG}_{\mathrm{LDSDm}}\right)$ and the proportion of low- and high-density genotyped females was set to 0.05 and 0 , respectively. Each graph in Figure 2 represents a given $\mathrm{PG}_{\mathrm{LD}, \mathrm{SDm}}$ value, where only graphs with an underlying $\mathrm{PG}_{\mathrm{LD}, \mathrm{SDm}}$ of $0(\Delta), 0.05(+)$, and $0.95(\diamond)$ are marked.

$\mathrm{PG}_{\mathrm{HD}, \mathrm{SDm}}$ were always at a level where they could be decreased without a major loss in $\Delta \mathrm{G}_{\mathrm{i}}$. In contrast, a decrease in the already low $\mathrm{PG}_{\mathrm{LD}, \mathrm{DS}}$ led, in turn, to a lower $\Delta \mathrm{G}_{\mathrm{DS}}$. As a consequence, an increase in $\mathrm{C}_{\mathrm{GEBV}}$ resulted in 2 different scenarios: A) the increase was so high that even refraining from genotyping bulls did not save enough money to maintain the level of genotyping in the path DS and the proportion of genotyped dams had to be decreased; the available funds were used to increase the genotyping of males to maximize the $\Delta \mathrm{G}_{\mathrm{SDm}}$; $\left.\mathrm{B}\right)$ The increase in $\mathrm{C}_{\mathrm{GEBV}}$ was so high that a decrease in the number of genotyped individuals in path SDm saved enough money to maintain the level of genotyping in path DS; because this decrease barely affected $\Delta \mathrm{G}_{\mathrm{SD}}$ due to an already high selection intensity, GS in path DS was maintained at the given level and $\Delta \mathrm{G}_{\mathrm{a}}$ was only slightly reduced. Scenario A displays the positive effect of $\mathrm{C}_{\mathrm{GEBV}, \mathrm{LD}}$ on $\Delta \mathrm{G}_{\mathrm{SDm}}$ and scenario $\mathrm{B}$ explains the strong effect of $\mathrm{C}_{\mathrm{GEBV}}$ on $\mathrm{PG}_{\mathrm{HD}, \mathrm{SDm}}$ and $\mathrm{PG}_{\mathrm{LD}, \mathrm{SDm}}$.

\section{Sensitivity of Maximization Results to Changes in Accuracy and Cost}

Any change of the relevant input parameters would require a reorganization of the breeding scheme in terms of adjusting $\mathrm{PG}_{\mathrm{HD}, \mathrm{i}}$ and $\mathrm{PG}_{\mathrm{LD}, \mathrm{i}}$ to keep $\Delta \mathrm{G}_{\mathrm{a}}, \Delta \mathrm{G}_{\mathrm{SDm}}$, and $\Delta \mathrm{G}_{\mathrm{DS}}$ at their maximum values. The extent of this reorganization process is reflected by the similarity as a function of a change in a single input parameter between breeding scheme structures before and after reorganization. Each subdiagram in Figure 3 visualizes the development of the mean of this similarity as a function of a change of 1 input parameter. The underlying values are calculated between optimum basic schemes and optimum schemes differing from these only by a single input parameter (the basic parameter), whose variation is reflected on the $\mathrm{x}$-axis of each subdiagram. For example, Figure 3b reflects the similarity as a function of $\mathrm{C}_{\mathrm{GEBV}, \mathrm{LD}}$. Basic schemes of each subdiagram are characterized by the minimum of the basic parameter. For instance, $\mathrm{C}_{\mathrm{GEBV}, \mathrm{LD}}$ of all basic schemes is equal to its minimum, $20 €$. The number of basic schemes is 180 (range of $\mathrm{C}_{\mathrm{GEBV}, \mathrm{HD}} \times$ range of $\mathrm{r}_{\mathrm{GEBV}, \mathrm{HD}} \times$ range of $\left.\mathrm{r}_{\mathrm{GEBV}, \mathrm{LD}}\right)$. The similarity plotted at $\mathrm{C}_{\mathrm{GEBV}, \mathrm{LD}}=40$ is the mean of the similarity of each of the 180 basic schemes to those 180 schemes with which they have all input parameters in common except $\mathrm{C}_{\mathrm{GEBV}, \mathrm{LD}}$. The slope of the mean curve can be seen as an indicator for the sensitivity of the breeding scheme structures to a change of a single input parameter value.

In general, changes in $\mathrm{r}_{\mathrm{GEBV}, \mathrm{LD}}$ and $\mathrm{C}_{\mathrm{GEBV}, \mathrm{LD}}$ imposed the highest pressure on breeding scheme structures to maintain a high $\Delta \mathrm{G}_{\mathrm{a}}$. In both cases, the similarity decreased below 0.4 , whereas similarity remained above 0.7 over the range of variation in $\mathrm{r}_{\mathrm{GEBV}, \mathrm{HD}}$ and $\mathrm{C}_{\mathrm{GEBV}, \mathrm{HD}}$. Furthermore, if $\mathrm{C}_{\mathrm{GEBV}, \mathrm{HD}}$ increased from $190 €$ to 210 $€$ per genotyped individual, the structure of breeding schemes became more similar to the basic scheme. This indicates a certain threshold at which a further increase 
(a) High-density SNP chips

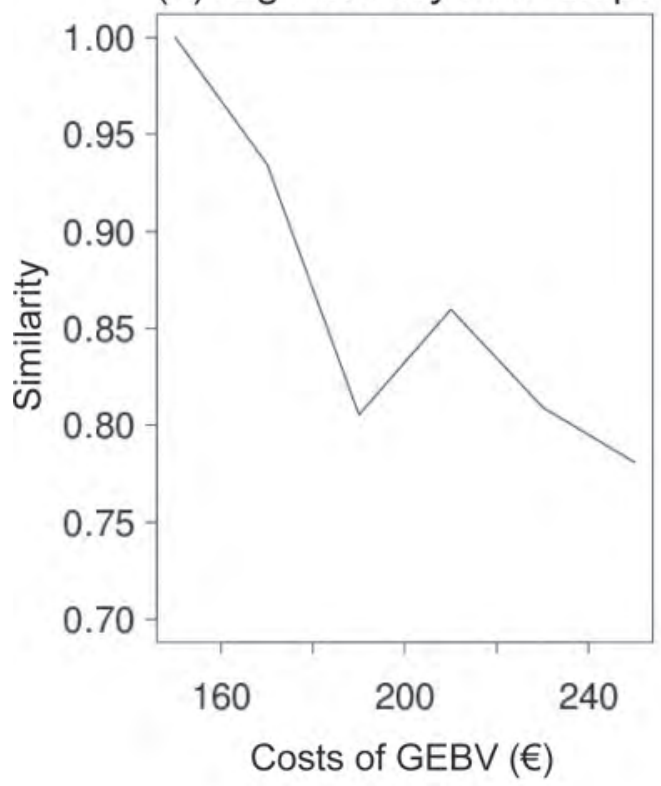

(c) High-density SNP chips

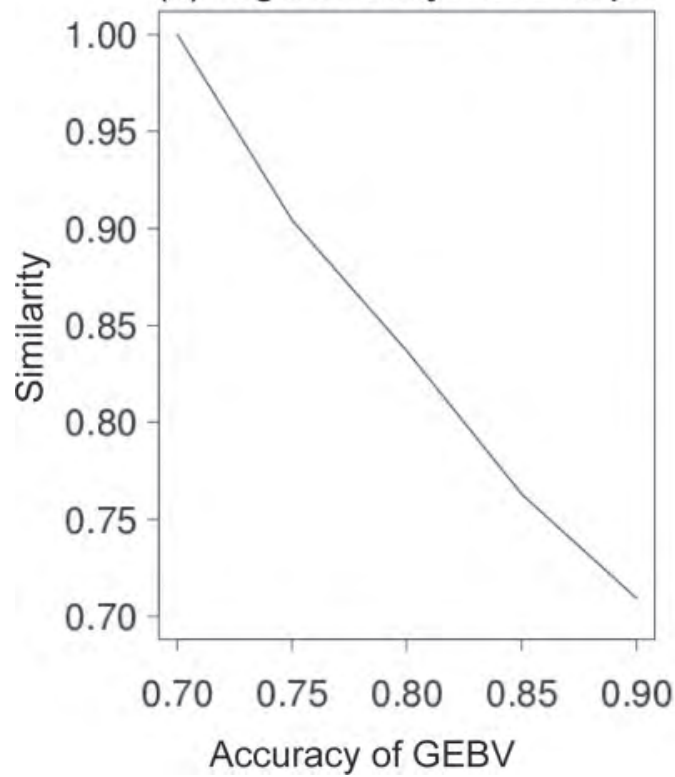

(b) Low-density SNP chips

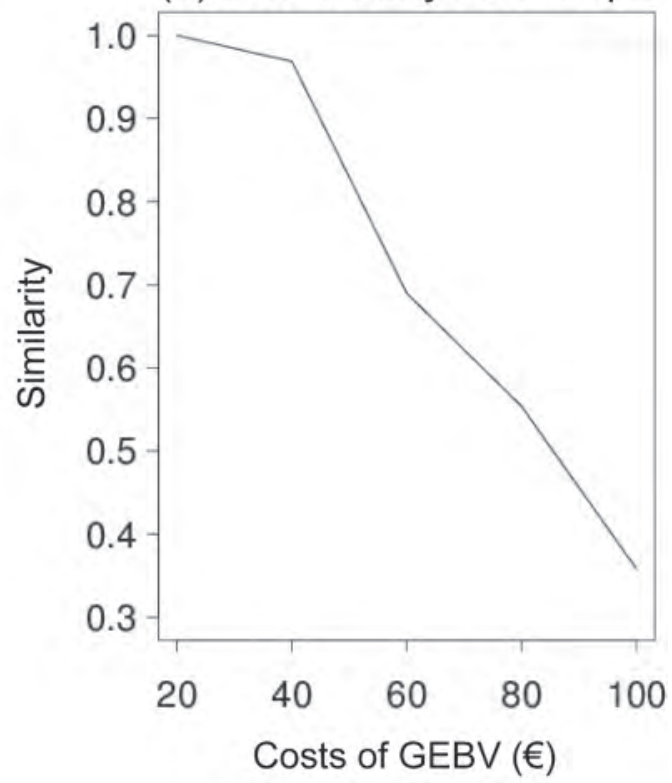

(d) Low-density SNP chips

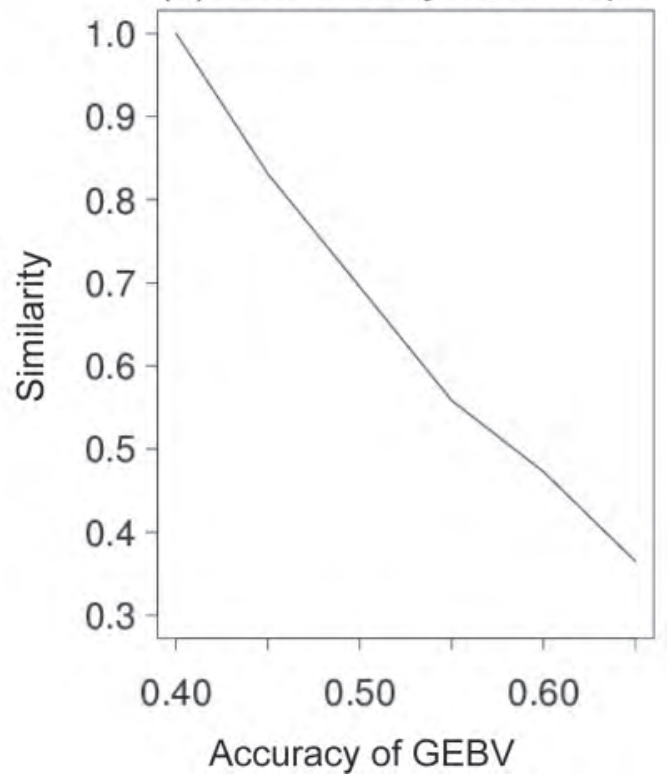

Figure 3. The structural similarity (correlation) between breeding schemes. The similarity graph given in each subdiagram was calculated as the mean of the similarities between breeding schemes maximizing the genetic gain per year $\left(\Delta \mathrm{G}_{\mathrm{a}}\right)$ for the minimum of the $\mathrm{x}$-axis parameter (the basic schemes), and breeding schemes maximizing $\Delta \mathrm{G}_{\mathrm{a}}$ for input parameters set equal to those of the basic schemes but different in the $\mathrm{x}$-axis parameter. This difference is equal to the variation steps of the $\mathrm{x}$-axis parameter. GEBV $=$ genomic EBV.

in $\mathrm{C}_{\mathrm{GEBV}, \mathrm{HD}}$ could not be compensated for by a reduction of $\mathrm{PG}_{\mathrm{HD}, \mathrm{SDm}}$ without a major loss in $\Delta \mathrm{G}_{\mathrm{a}}$. As a consequence, the proportion of genotyped females was decreased, allowing for an increased number of genotyped males, which in turn, led to a higher similarity. Referring to the scenarios outlined above to maintain a high genetic gain under increased genotyping cost, the large effect of $\mathrm{C}_{\mathrm{GEBV}, \mathrm{LD}}$ and $\mathrm{r}_{\mathrm{GEBV}, \mathrm{LD}}$ is explained by the same mechanism.

\section{DISCUSSION}

Several authors found the use of low-density SNP chips in combination with imputation algorithms to 
be a useful strategy for applying genomic selection to species where females have low reproductive capacities (Habier et al., 2009; VanRaden, 2010). In dairy cattle, this might be especially the case for selecting bull dams, as the relatively low number of annually recruited cow and bull sires, and the advantages from the shortened generation interval justify even high expenses for genotyping males. One of the major difficulties for the application of GS in the DS path is the necessity of a large number of dams for producing male selection candidates. This makes high GS intensity almost impossible if the cost per individual is high relative to the available budget. This might also be the case when using reproductive techniques to increase the number of male calves per cow, thereby decreasing the number of necessary bull dams and increasing the selection intensity. The cost of such techniques may exhaust the economic resources as well as increase the number of genotyped individuals. Because cheap low-density SNP chips allow for genotyping large numbers of potential bull dams, a GEBV with a sufficient accuracy may generate a higher genetic gain compared with using expensive high-density SNP chips. This assumption is supported by the outcomes of this study, which clearly shows that the selection path dam-sire is the major application for low-density genotyping.

In this study, even for low $\mathrm{r}_{\mathrm{GEBV}, \mathrm{HD}}$ of 0.7 and $\mathrm{r}_{\mathrm{GEBV}, \mathrm{LD}}$ of 0.4 we found a $\Delta \mathrm{G}_{\mathrm{a}}$ of 0.47 genetic standard deviations. This is higher than other published results assuming higher accuracies of GEBV (Schaeffer, 2006; Lillehammer et al., 2011), and might be due to the very high selection intensities for bull sires and cow sires in our calculations and a minimum $\mathrm{r}_{\mathrm{GEBV}, \mathrm{HD}}$ of 0.7. Without regarding costs, Schaeffer (2006) found the selection path DS to be the most important under the assumption that GEBV are available for the whole cow population and that they are as accurate as GEBV for selecting male calves. Under restricted costs, however, this might be not possible due to a limited number of genotyped potential bull dams. As in our calculations, genotyping of all potential bull dams was impossible because of cost limitations. Even when using very cheap low-density SNP chips, $\Delta \mathrm{G}_{\mathrm{DS}}$ never exceeded $\Delta \mathrm{G}_{\mathrm{SS}}$ or $\Delta \mathrm{G}_{\mathrm{SDm}}$. Furthermore, in the same article, a large decrease in breeding cost was predicted. Contrary to that suggestion, we found that expanding the number of genotyped potential bull dams to be the most important cost driver. Thus, disproportionate genotyping should be avoided in this selection path, and instead, a sound multistage selection should be used.

Genetic variance is known to be reduced by selectioninduced gametic disequilibrium (Bulmer, 1971), leading to an overestimation of asymptotic rates of genetic response (Wray and Hill, 1989; Dekkers, 1992; Villanueva et al., 1993) if this effect is not accounted for, as in our study. Ranking and relative differences between alternative breeding programs have, however, been found to be little affected by ignoring this effect (Wray and Hill, 1989; Dekkers, 1992). Rates of inbreeding - though not evaluated in detail - should be comparable between the schemes considered, because of the fixed number breeding animals eventually selected.

Due to the nonlinearity of the genetic gain as a function of the selection intensity or the proportion selected, we found that a 3-stage selection of bull dams on the basis of pedigree information, $\mathrm{GEBV}_{\mathrm{LD}}$, and $\mathrm{GEBV}_{\mathrm{HD}}$ was the optimum strategy in terms of $\Delta \mathrm{G}_{\mathrm{a}}$ in more than half of all parameter combinations. Thus, if a sufficiently accurate pedigree preselection takes place, a level of $\mathrm{GS}_{\mathrm{LD}}$ above a certain value will generate less $\Delta \mathrm{G}_{\mathrm{a}}$ than using an additional $\mathrm{GS}_{\mathrm{HD}}$ stage with low selection intensity. Due to such multistage selection procedures, a given $\Delta \mathrm{G}_{\mathrm{i}}$ in a certain selection path can be achieved at lower cost, or a higher $\Delta \mathrm{G}_{\mathrm{a}}$ can be achieved at the same expenses by allocating investments to selection paths according to the marginal benefit of additional selection intensity. This is exemplified by the usage of $\mathrm{GS}_{\mathrm{LD}}$ for selecting male calves in combination with $\mathrm{GS}_{\mathrm{HD}}$, being only a result of the possible cost reductions in the path SDm, and not of a higher $\Delta \mathrm{G}_{\mathrm{SDm}}$ due to including another information source in the selection process. The available funds could then be used in the path DS to increase the $\mathrm{GS}_{\mathrm{LD}}$ selection intensity, or to implement an additional $\mathrm{GS}_{\mathrm{HD}}$ stage, leading to an increased $\Delta \mathrm{G}_{\mathrm{DS}}$ and a higher $\Delta \mathrm{G}_{\mathrm{a}}$. These outcomes are in contrast to ideas of genotyping even whole subpopulations to identify individuals with the highest GEBV (König and Swalve, 2009).

The difficulty of genomic selection to generate a sufficient $\Delta \mathrm{G}_{\mathrm{DS}}$ under the constraint of a limited budget is the major force behind the sensitivity of the structure of the breeding schemes to changes in 1 or more input parameters. Thus, breeding companies implementing excessively low-density genotyping may be forced to adjust their selection procedure if genotyping costs increase. Although one may consider this an unlikely scenario, the large number of genotyped females necessary to implement GS in this selection path makes breeding companies very susceptible to any changes in genotyping costs. Furthermore, GS of females might lack the economic justification when comparing financial efforts and $\Delta \mathrm{G}_{\mathrm{DS}}$. Finally, even in the path SDm, breeding costs can be decreased if an economic optimum in terms of comparing the marginal benefit of increased selection intensity and the related financial efforts is found. Breeding companies exploiting this optimum might gain advantage from selling their products at lower prices. 


\section{CONCLUSIONS}

The results of our study showed that low-density SNP chips may be particularly useful for extensive genotyping of potential bull dams in dairy cattle breeding programs. This even holds if accuracies of GEBV are low. Limitation of the available budget can lead to strong interdependencies between optimum selection measures within male and female selection paths. The reasons are decreasing marginal benefits from increasing selection intensities, in particular selection paths accompanied by increased opportunity cost due to limited response in other paths. Optimum allocation of financial resources leads to multistage GS. Breeding organizations redesigning their breeding schemes to capitalize on genomic selection will have to take these interdependencies into account.

\section{ACKNOWLEDGMENTS}

This work was financed by the German Federal Ministry for Education and Research (BMBF, Berlin, Germany), project "FUGATO-PLUS Brain 0315136D." Many thanks to Heiko Güldenpfenning (Rinderzucht Mecklenburg-Vorpommern, Woldegk, Germany) for providing economic data.

\section{REFERENCES}

Brent, R. P. 1973. Algorithms for Minimization without Derivatives. Prentice-Hall, Englewood Cliffs, NJ.

Bulmer, M. 1971. The effect of selection on genetic variability. Am. Nat. 105:201-211. http://dx.doi.org/10.1086/282718.

Calus, M. P. L., and R. F. Veerkamp. 2007. Accuracy of breeding values when using and ignoring the polygenic effect in genomic breeding value estimation with a marker density of one SNP per cM. J. Anim. Breed. Genet. 124:362-368. http://dx.doi.org/10.1111/ j.1439-0388.2007.00691.x.

Cochran, W. 1951. Improvement by means of selection. Pages 449-470 in Proc. 2nd Berkeley Symposium on Mathematical Statistics and Probability, Berkeley, CA. University of California Press, Berkeley.

Daetwyler, H. D., B. Villanueva, P. Bijma, and J. A. Woolliams. 2007. Inbreeding in genome-wide selection. J. Anim. Breed. Genet. 124:369-376. http://dx.doi.org/10.1111/j.1439-0388.2007.00693.x.

Dekkers, J. C. M. 1992. Asymptotic response to selection on best linear unbiased predictors of breeding vales. Anim. Prod. 54:351360. http://dx.doi.org/10.1017/S0003356100020808.

Dekkers, J. C. M. 2007. Prediction of response to marker-assisted and genomic selection using selection index theory. J. Anim. Breed. Genet. 124:331-341. http://dx.doi.org/10.1111/j.14390388.2007.00701.x.

Genz, A. 1992. Numerical computation of multivariate normal probabilities. J. Comput. Graph. Statist. 1:141-149. http://dx.doi. org $/ 10.2307 / 1390838$.

Habier, D., R. L. Fernando, and J. C. M. Dekkers. 2009. Genomic selection using low-density marker panels. Genetics 182:343-353. http://dx.doi.org/10.1534/genetics.108.100289.

Hayes, B. J., P. J. Bowman, A. J. Chamberlain, and M. E. Goddard. 2009. Invited review: Genomic selection in dairy cattle: Progress and challenges. J. Dairy Sci. 92:433-443. http://dx.doi. org/10.3168/jds.2008-1646.
König, S., and H. H. Swalve. 2009. Application of selection index calculations to determine selection strategies in genomic breeding programs. J. Dairy Sci. 92:5292-5303. http://dx.doi.org/10.3168/ jds.2009-2232.

Lillehammer, M., T. Meuwissen, and A. Sonesson. 2011. A comparison of dairy cattle breeding designs that use genomic selection. J. Dairy Sci. 94:493-500. http://dx.doi.org/10.3168/jds.2010-3518.

Lynch, M., and B. Walsh. 1998. Genetics and Analysis of Quantitative Traits. Sinauer Associates Inc., Sunderland, MA.

Meuwissen, T. H., B. J. Hayes, and M. E. Goddard. 2001. Prediction of total genetic value using genome-wide dense marker maps. Genetics 157:1819-1829.

Mi, X., and F. Utz. 2008. R-Package selection gain. University of Hohenheim. http://cran.r-project.org/web/packages/selectiongain/ index.html.

Muir, W. M. 2007. Comparison of genomic and traditional BLUPestimated breeding value accuracy and selection response under alternative trait and genomic parameters. J. Anim. Breed. Genet. 124:342-355. http://dx.doi.org/10.1111/j.1439-0388.2007.00700.x.

Namkoong, G. 1970. Optimum allocation of selection intensity in two stages of truncation selection. Biometrics 26:465-476. http:// dx.doi.org/10.2307/2529102.

Rendel, J. M., and A. Robertson. 1950. Estimation of genetic gain in milk yield by selection in a closed herd of dairy cattle. J. Genet. 50:1-8. http://dx.doi.org/10.1007/BF02986789.

Schaeffer, L. R. 2006. Strategy for applying genome-wide selection in dairy cattle. J. Anim. Breed. Genet. 123:218-223. http://dx.doi. org/10.1111/j.1439-0388.2006.00595.x.

Tallis, G. M. 1961. The moment generating function of the truncated multi-normal distribution. J. R. Stat. Soc., B 23:223-229.

VanRaden, P. 2010. Genomic evaluations with many more genotypes and phenotypes. Abstract 0027 in Proc. 9th World Congr. Genet. Appl. Livest. Prod., Leipzig, Germany. German Society for Animal Science, Leipzig, Germany.

Villanueva, B., N. Wray, and R. Thompson. 1993. Prediction of asymptotic rates of response from selection on multiple traits using univariate and multivariate best linear unbiased predictors. Anim. Prod. 57:1-13. http://dx.doi.org/10.1017/S0003356100006541.

Weigel, K., G. de los Campos, A. Vazquez, C. V. T. G. Rosa, D. Gianola, J. O'Connell, P. VanRaden, and G. Wiggans. 2010. Genomic selection and its effects on dairy cattle breeding programs. Abstract 0119 in Proc. 9th World Congr. Genet. Appl. Livest. Prod., Leipzig, Germany. German Society for Animal Science, Leipzig, Germany.

Wray, N., and W. G. Hill. 1989. Asymptotic rates of response from index selection. Anim. Prod. 49:217-227. http://dx.doi. org/10.1017/S0003356100032347.

\section{APPENDIX}

According to path coefficient methodology (Lynch and Walsh, 1998) and the approach of Dekkers (2007), a certain phenotype $P$ can be decomposed as follows:

$$
\begin{aligned}
& P=G+E \\
& G=Q+R \\
& Q=\hat{Q}_{i}+e_{i} \\
& \hat{Q}_{i}=\hat{Q}_{j}+e_{j} \\
& P=E+R+e_{i}+\hat{Q}_{j}+e_{j},
\end{aligned}
$$

where $G$ is the additive genetic effect, $E$ is the random environmental effect, $Q$ is the true marker breeding value, $R$ is the polygenic effect not explainable by the markers, $\hat{Q}_{i}$ is estimated marker breeding value using a 
high-density SNP chip, $e_{i}$ is the error due to estimating the marker effects, $\hat{Q}_{j}$ is the estimated marker breeding value using a low-density SNP chip and imputation algorithms, and $e_{j}$ is the imputation error.

Assuming a 0 covariance between $\hat{Q}_{j}$ and $e_{j}$, the correlation between $\hat{Q}_{i}$ and $\hat{Q}_{j}$ is

$$
r_{\left(\hat{Q}_{i} \hat{Q}_{j}\right)}=\frac{\sigma_{\left(\hat{Q}_{j}+e_{j}, \hat{Q}_{j}\right)}}{\sigma_{\left(\hat{Q}_{j}+e_{j}\right)} \sigma_{\left(\hat{Q}_{j}\right)}}=\frac{\sigma_{\left(\hat{Q}_{j}\right)}^{2}}{\sigma_{\left(\hat{Q}_{i}\right)} \sigma_{\left(\hat{Q}_{j}\right)}}=\frac{\sigma_{\left(\hat{Q}_{j}\right)}}{\sigma_{\left(\hat{Q}_{i}\right)}} .
$$

The latter formula assumes - as a consequence of chip design - that all variance in the predictions from the LD chip is also captured by the HD chip, yet the variance of the predictions from the $\mathrm{LD}$ chip is smaller. If this is not the case, the covariance in Equation 11 will be smaller than both standard deviations in the denominator, resulting in a lower correlation. 